seilles). This considered the use of thermionic solar energy convertors. Solar energy was not used merely to heat the electron emitter, but also used for neutralizing the space charge by the ionization of caesium vapour in the space between the electrodes.

A. VЕСHT

\section{BRITTLE FRACTURE}

THE brittle fracture of ceramics, glasses, metals and amorphous glassy polymers (plastics) was the subject of a meeting held at the Rubber and Plastics Research Association, Shawbury, Shrewsbury, Shropshire, on December 6 , 1965. The title was chosen to indicate a concern for the behaviour of real materials rather than the classical theoretical models. The discussion was divided into four parts, each devoted to one material, and the significant features of the fracture behaviour of each of them were reviewed by four main speakers-Dr. F. J. P. Clarke, A.E.R.E., Harwell (Ceramies); Dr. D. M. Marsh, Tube Investments Research Laboratories, Saffron Walden (Glasses); Prof. K. E. Puttick, Battersea College of Technology (Metals); and Dr. J. P. Berry, Rubber and Plastics Research Association, Shawbury (Plastics).

It was clearly desirable to define or at least to clarify the basic concepts of the subject, but there were marked differences of point of view and opinion. No universally acceptable definition of "brittle fracture" could be formulated and it was clear that the more complex the mechanical response of a material, the more difficult it is to describe precisely its failure characteristics. For metals, which caused the greatest controversy at the meeting, it was concluded that instead of seeking a precise definition, behaviour should be described in terms of specific characteristics such as the micromode of failure, crack stability, local deformation and the like.

The theoretical foundation for much of the discussion was the energy balance approach of Griffith, but here too there was some difference in the interpretation of the theory, particularly between the physical scientists and the engineers. The former were concerned with the relation of the theory to the detailed mechanism of failure, and the latter with its relation to the stability of engineering structures. This led to the question, not completely rosolved, of whether the condition given by the theory is both necessary and sufficient for fracture.

The aspects of fracture common to all the materials are (i) initiation and the nature of the primary flaw, (ii) the fracture surface energy, and (iii) crack propagation.

Initiation and the primary flaw. Contrary to the simple Griffith model, the flaw which is responsible for failure in ceramics, plastics and metals is not present as such in the unstressed material. In ceramics the mismatch in expansion coefficient at grain boundaries produces local concentrations of stress which can result in the formation of micro-cracks at applied loads less than those required for fracture. Similarly, in glassy plastics the crazes produced at relatively low stresses result in failure at the ultimate stress and, in metals, flaws are produced by slip or twinning processes. The details of the flaw generation process are different in the three cases, however. Ceramics have little capacity for plastic flow and little energy is dissipated in forming the flaws. In plastics the craze structure is complex; it represents a region of local yielding under hydrostatic tension and the structural changes that occur result in void formation and molecular orientation. Plastic flow is intimately concerned in the formation of the primary flaw in metals.

In glasses the flaws probably arise adventitiously, by mechanical damage, and correspond more closely to the primary flaw as defined in the Griffith theory.

Fracture surface energy. The application of the Griffith criterion to the fracture of a material yields a value for the energy required for the formation of unit area of fracture surface. For all the materials the energy, determined experimentally, is much greater than the true surface free energy, and the discrepancy can be accounted for by $(a)$ the non-planar character of the fracture surface, (b) the formation of subsurface flaws, and (c) inelastic processes occurring at the fracture plane. All three factors have been considered in explaining the behaviour of ceramics but it is not yet possible to assess their relative contributions. In plastics the discrepancy is believed to be due to structural changes at the fracture surface which can be detected optically. Similarly the discrepancy in metals is attributed to the plastics flow processes occurring at the fracture plane. Only recently has it been shown conclusively that the fracture surface energy of glass is much greater than the theoretical value. Indentation experiments indicate that glass can deform inelastically and the discrepancy of surface energy may be due to this. Indirect evidence indicates that the zone in which the energy is dissipated extends only $60 \AA$ from the fracture plane.

Crack propagation. The Griffith theory implies that the application of a certain eritical stress will cause crack extension and fracture. The behaviour of real materials is much more complex. In ceramics, the surface irregularity and hence the fracture surface energy increases with the length of the crack and it is not obvious which combination of the two factors corresponds to the onset of catastrophic crack propagation. The initial rate of growth of a crack in plastics is low, but after travelling a short distance it suddenly and discontinuously increases. The reason for this is not known, but it may again be the result of the dependence of fracture surface energy on crack length. The similar change in crack velocity observed in metals is considered to involve a ductile brittle transition at a particular size of yielded region, possibly bccause of increasing triaxiality of the stress at the crack tip. The plastic zone at the tip of a crack travelling in glass is much smaller, and no change in velocity or surface configuration corresponding to a transition from brittle to ductile has been detected. Its influence, however, is felt in reducing the terminal velocity below its theoretical value.

At the meeting there were many points of similarity and difference in the behaviour of the materials under review and they were of particular interest when they were unexpected, as were some similarities of the behaviour of materials of different structure, and some differences of behaviour of similar materials. Observations on one material frequently suggested that interesting results may be obtained from similar experiments on other materials or by searching for effects and correlations which have not previously been considered. In this respect the meeting fulfilled its objective, and, it is hoped, supplied the stimulus for further studies to provide a better understanding of the complex phenomenon of brittle fracture.

J. P. BERRY

(Continued from page 13)

national scientific co-operation provides a basis for developing effective contacts between nations with widely difforent political systems. In such programmes, all ultimately depends on the willingness of individual nations and the energy and enthusiasm of their individual scientists. In the Antarctic, now as in the earlier "heroic age" of exploration, all achievement likewise depends on the energy, enthusiasm, and willingness to co-operato of each individual member of the national and international team. Judged against the direct record of research achievement and the indirect by-products of international amity and understanding, Antaretic science over the past decade has been an outstandingly good investment. 